free legislation, the persisting relative inequalities in SHS exposure by SES highlight the need for continued investment in tobacco control policies.

\section{OP16 DEVELOPING A SMOKE-FREE HOME INTERVENTION FOR NEONATAL INTENSIVE CARE UNITS - A QUALITATIVE STUDY}

${ }^{1} \mathrm{CJ}$ Notley*, ${ }^{1} \mathrm{TJ}$ Brown, ${ }^{2} \mathrm{~A}$ Nichols, ${ }^{3} \mathrm{~L}$ Bauld, ${ }^{4} \mathrm{~W}$ Hardeman, ${ }^{5} \mathrm{E}$ Boyle, ${ }^{5} \mathrm{M}$ Hubbard ${ }^{4} \mathrm{~F}$ Naughton, ${ }^{6} \mathrm{M}$ Ussher, ${ }^{1,2} \mathrm{P}$ Clarke, ${ }^{7} \mathrm{R}$ Holland, ${ }^{8} \mathrm{~S}$ Orton. ${ }^{1}$ Norwich Medical School, University of East Anglia, Norwich, UK; ${ }^{2}$ Norfolk and Norwich University Hospitals NHS Foundation Trust, Norwich, UK; ${ }^{3}$ Edinburgh University, Edinburgh, UK; ${ }^{4}$ School Of Health Sciences, University of East Anglia, Norwich, UK; ${ }^{5}$ University Hospitals of Leicester NHS Trust, Leicester, UK; ${ }^{6}$ St Georges, University of London and University of Stirling, London and Stirling, UK; ${ }^{7}$ Leicester Medical School, Leicester, UK; ${ }^{8}$ University of Nottingham, Nottingham, UK

\subsection{6/jech-2020-SSMabstracts. 16}

Background Babies born to smokers weigh on average $200 \mathrm{~g}$ less than those born to non-smokers and are at $40 \%$ higher risk of being born preterm. The relative risk of admission to Neonatal Intensive Care units (NICU) for infants of smokers is increased by at least $20 \%$. Parents of infants admitted to NICU may feel helpless and overwhelmed at a time when their baby is critically ill. Stopping smoking, or remaining abstinent, is one of the few things that parents can do to significantly improve the longer-term recovery and health of their offspring, yet stressed parents are at increased risk of smoking relapse. NICU admission may represent a 'teachable moment' where parents are receptive to smoking cessation.

Methods Qualitative focus groups and interviews with parents and family members of babies admitted to NICUs. Participants were purposively sampled $(n=60)$ from NICUs across two large UK teaching hospitals, seeking maximum variation in smoking status, parental/familial status, ethnicity and socioeconomic status. Qualitative topic guides sought feedback on potential intervention approaches, considering 'who' might introduce, 'what' might be the content, and 'when' an intervention might be delivered. Data were collected face to face by dedicated neonatal research nurses. All data were audio recorded and transcribed verbatim. Inductive thematic analysis of data was conducted by two members of the research team, independently reviewing coding to reach consensus on emergent themes.

Results Parents appear amenable to smoking cessation and express surprise that the subject is not addressed. Immediate addressing of smoking status would not be appropriate on acute admission to NICU due to stress and concerns regarding the newborn, but timely support is needed to reach those willing to quit, and those who had quit during pregnancy but were at high risk of relapse. Support might best be delivered by a NICU nurse with specialist training. Support with cessation and relapse prevention through information about smokefree homes, nicotine replacement therapy and/or support to use nicotine in significantly less harmful ways (e.g. vaping) were identified as promising routes for intervention. Parents welcomed ongoing support following discharge from NICU and were amenable to digital options.

Conclusion There is presently little dedicated support for smoking cessation, relapse prevention or smoke-free homes for families of NICU babies. Parents are amenable to support and consider a focus on smoke-free homes as a less stigmatising way in which smoking may be discussed and cessation promoted to improve the health of premature babies.

\section{OP17 EXAMINING INEQUALITY IN TRIALS OF SMOKING CESSATION INTERVENTIONS DELIVERED IN PRIMARY CARE: CRITIQUE AND REANALYSIS OF COCHRANE REVIEWS}

\author{
1,2JM Birch*, ${ }^{2} \mathrm{H}$ Dambha-Miller, ${ }^{1,2}{ }^{2} \mathrm{SJ}$ Griffin, ${ }^{2} \mathrm{~GB}$ Hutton, ${ }^{2} \mathrm{MP}$ Kelly, ${ }^{2} \mathrm{AL}$ Kinmonth. ${ }^{1} \mathrm{MRC}$ \\ Epidemiology Unit, University of Cambridge, Cambridge, UK; ${ }^{2}$ Department of Public Health \\ and Primary Care, University of Cambridge, Cambridge, UK

\subsection{6/jech-2020-SSMabstracts. 17}

Background Tobacco smoking is a major cause of chronic disease and premature mortality. Its effects are socially patterned. Observational studies show that low socioeconomic status [SES] is associated with higher smoking prevalence and lower cessation rates. Interventions in primary care may improve or exacerbate health inequalities depending on socioeconomic patterning of access and uptake. Data on the impact of trials of smoking cessation interventions delivered in primary care on health inequalities by SES have not been synthesised. We examined the impact of smoking cessation interventions delivered in primary care on inequalities in health by socioeconomic status.

Methods We searched the Cochrane database of systematic reviews from inception until June 2019. We included reviews of trials of smoking cessation interventions delivered in primary care and published in English.

Results We identified eight Cochrane reviews (413 studies). Eighty five studies included an intervention delivered in primary care. Interventions were: behavioural, (very) brief advice, and pharmacological (including nicotine replacement therapy). Full texts were accessed for 70 studies; 17 reported an SES measure. Two studies targeted low-SES groups. There was heterogeneity in SES measures used across the studies, which included household income, occupational level and social class. Three studies analysed SES as a predictor of effectiveness of the smoking cessation intervention; none found that effectiveness differed by SES.

Discussion This summary and critique of Cochrane reviews demonstrates that trials of smoking cessation interventions delivered in primary care are not designed to allow analysis of effects by measures of SES. Studies rarely reported SES of participants at baseline and hardly ever as a predictor of smoking cessation. Our work highlights the need for routine reporting of SES amongst trials and greater consensus in included measures. Consistent reporting of a core set of SES indicators will enable testing of similarities between trial groups and differential effects by SES.

\section{OP18 FROM SMOKING-PERMITTED TO SMOKEFREE PRISONS: A 3-YEAR EVALUATION OF THE CHANGES IN OCCUPATIONAL EXPOSURE TO SECOND-HAND SMOKE ACROSS A NATIONAL PRISON SYSTEM}

${ }^{1} S$ Semple*, ${ }^{2} E$ Demou, ${ }^{1} \mathrm{R}$ Dobson, ${ }^{2} \mathrm{H}$ Sweeting, ${ }^{3} \mathrm{~S}$ Sidwell, ${ }^{1} \mathrm{~A}$ Brown, ${ }^{1} \mathrm{R}$ O'Donnell, ${ }^{1} \mathrm{~K}$ Hunt. ${ }^{1}$ Institute for Social Marketing and Health, University of Stirling, Stirling, UK; ${ }^{2} \mathrm{MRC}$ CSO SPHSU, University of Glasgow, Glasgow, UK; ${ }^{3}$ Scottish Prison Service, SPS, Edinburgh, UK

\subsection{6/jech-2020-SSMabstracts. 18}

Background Prisons were one of the only workplaces where smoking continued to be permitted after the smoking ban in indoor public places in Scotland in 2006. Hence, the prison workforce remained potentially exposed to secondhand smoke 